\title{
Comparative Transcriptome Analysis Identifies Crucial Candidate Genes and Pathways in The Hypothalamic-Pituitary-Gonadal Axis During External Genitalia Development of Male Geese
}

\section{Bincheng Tang}

Sichuan Agricultural University

Shenqiang $\mathrm{Hu}$

Sichuan Agricultural University

Qingyuan Ouyang

Sichuan Agricultural University

Tianhao Wu

Sichuan Agricultural University

Yao Lu

Sichuan Agricultural University

Jiwei Hu

Sichuan Agricultural University

Bo Hu

Sichuan Agricultural University

Liang Li

Sichuan Agricultural University

Jiwen Wang ( $\nabla$ wjw2886166@163.com )

Sichuan Agricultural University

\section{Research Article}

Keywords: male goose, HPG axis, external genitalia development, transcriptome sequencing

Posted Date: October 5th, 2021

DOl: https://doi.org/10.21203/rs.3.rs-892387/v1

License: (1) This work is licensed under a Creative Commons Attribution 4.0 International License. Read Full License 


\section{Abstract \\ Background}

All birds reproduce via internal fertilization, but only $~ 3 \%$ of male birds possess the external genitalia that allows for intromission. Waterfowl (e.g., duck and goose) are representatives of them, and the external genitalia development of male geese is directly related to mating ability. Notably, some male geese show abnormal external genitalia development during ontogenesis. However, until now little is known about the molecular mechanisms of the external genitalia development in goose. In the present study, comparative transcriptomic analyses were performed on the hypothalamus, pituitary gland, testis, and external genitalia isolated from the 245-day-old male Tianfu meat geese showing normal (NEGG, $n=3$ ) and abnormal (AEGG, $n=3$ ) external genitals in order to provide a better understanding of the mechanisms controlling the development of the external genitalia in aquatic bird species.

\section{Results}

There were 107, 284, 2192, and 1005 differentially expressed genes (DEGs) identified in the hypothalamus, pituitary gland, testis and external genitalia between NEGG and AEGG. Functional enrichment analysis indicated that the DEGs identified in the hypothalamus were mainly enriched in the ECM-receptor interaction pathway. The ECM-receptor interaction, focal adhesion, and neuroactive ligandreceptor interaction pathways were significantly enriched by the DEGs in the pituitary gland. In the testis, the DEGs were involved in the neuroactive ligand-receptor interaction, cell cycle, oocyte meiosis and purine metabolism. In the external genitalia, the DEGs were mainly involved in the metabolic, neuroactive ligand-receptor interaction and WNT signaling pathways. Additionally, through protein-protein interaction (PPI) network and co-expression network integrated analysis, fifteen genes involved in the neuroactive ligand-receptor interaction and WNT signaling pathways were identified, including KNG1, LPAR2, LPAR3, NPY, PLCB1, AVPR1B, GHSR, GRM3, HTR5A, FSHB, FSHR, WNT11, WNT5A, WIF1, and WNT7B, which are potentially crucial for the development of goose external genitalia.

\section{Conclusions}

To our knowledge, this study is the first comparing the hypothalamus, pituitary gland, testis, and external genitalia transcriptomes of male geese exhibiting normal and abnormal external genitals. Significantly, we identified the key DEGs involved in the neuroactive ligand-receptor interaction pathway which regulates the WNT signaling pathway through $P L C B 1$ to control the development of male goose external genitalia.

\section{Background}


The external genitalia of most male birds will be degenerated and even lost during evolution. All birds reproduce via internal fertilization, but only $\sim 3 \%$ of male birds retain the external genitalia capable of intromission [1]. In poultry, most of terrestrial fowl (e.g., chickens) lack the intromittent external genitalia [2], while waterfowl (e.g., ducks and geese) retain the well-developed external genitalia [3]. During the internal fertilization process, the male external genitalia inserts into the female's cloaca to deliver sperm [4]. In waterfowl, the length and structural characteristics (e.g., smooth and the percentage for coverage with spines and grooves) of the external genitalia have been shown to be correlated with the frequency of copulations [5]. Therefore, the external genitalia plays an indispensable role in fertilization, consequently affecting the insemination capacity of male geese [6].

Over the past few year, studies in both mammals and birds have identified some genes related to external genitalia development through investigations of their mRNA expression levels. For instance, the external genitalia was absent in mice containing a targeted deletion of sonic hedgehog (Shh) [7]. In marsupials, expression of insulin growth factor 1 (IGF1) was upregulated during the external genitalia growth and elongation [8]. Herrera et al. (2013) found that the evolutionary reduction of the external genitalia in birds occurred by de novo activation of cell death by bone morphogenetic protein 4 (Bmp4) in the genital tubercle [1]. In male geese, the 17a-hydroxylase/17, 20-lyase (CYP17) plays a key role during the external genitalia growth [9]. Nevertheless, the regulatory mechanism of male goose external genitalia development remains unclear.

The hypothalamic-pituitary-gonadal (HPG) axis is a coordinated neuroendocrine system that regulates the development of poultry reproductive system $[10,11]$. Recently, the emerging omic studies on the HPG axis have revealed candidate genes and pathways that may have important roles in regulating reproduction-associated traits in poultry. For example, transcriptome sequencing of the hypothalamus and pituitary gland from chickens with different egg numbers revealed an important role of the steroid hormone biosynthesis pathway in the regulation of egg production performance [12]. Meanwhile, the mTOR signaling and PI3K-Akt signaling pathways have also been identified by transcriptome sequencing of chickens showing egg production performance [13]. Additionally, the DEGs identified in the hypothalamus and pituitary gland may be involved in broodiness in female geese [11]. However, such studies have been scarcely carried out in male geese regarding the mechanisms controlling the external genitalia development.

Next-generation sequencing (NGS) technology has provided a powerful, highly reproducible and costefficient tool for transcriptomic studies $[14,15]$. To date, RNA-seq has been used for analysis of reproduction-associated traits in poultry, including laying performance [16], and gonadal development [17]. Nevertheless, the mRNA expression profiling of HPG axis regulating external genital development of male goose has not been reported. Therefore, this study aimed to compare and analyze the expression profiles of the hypothalamus, pituitary gland, testis, and external genitalia between NEGG and AEGG geese using RNA-sEq. These data were expected to help elucidate the molecular mechanisms regulating male goose external genitalia development. 


\section{Results}

\section{Morphological differences in the external genitalia between NEGG and AEGG}

As shown in Fig. 1, male geese in the NEGG group showed an elongated and coiled external genitalia with dermal spines (Fig. 1a), whereas those in the AEGG group had a smooth external genitalia (Fig. 1b). In addition, compared with the AEGG group, the length of external genitalia in NEGG group was significantly longer than that in AEGG group ( $p<0.01$, Fig. 1c), and its weight was significantly heavier than that in AEGG group $(p<0.05$, Fig. 1 d).

\section{Overview Of Transcriptome Sequencing}

A total of $635,434,075$ raw reads were obtained from 24 samples through sequencing. Each sample yielded 25,138,908 clean reads after strict filtering. The Q20 (percentage of reads with a Phred quality value $>20$ ) and Q30 (percentage of reads with a Phred quality value >C30) of the clean reads ranged from $97.18 \sim 97.63 \%$ and $92.83 \sim 93.63 \%$, respectively. The mapping rate of the 24 samples ranged from 74.32 83.64\% (Additional file 3: Table S2).

Identification of the DEGS in the hypothalamus, pituitary gland, testis and external genitalia between NEGG and AEGG

We identified 107 DEGs in hypothalamus, including 41 up-regulated and 66 down-regulated genes. In the pituitary gland, 284 DEGs were found, of which 97 were up-regulated and 187 were down-regulated. In the testis, 2192 DEGs were found, of them 176 were up-regulated and 2016 were down-regulated. In the external genitalia, 1005 DEGs were found, of them 394 were up-regulated and 611 were down-regulated (Fig. 2a). Venn-diagram analysis revealed that 1 DEG displayed differential expression between hypothalamus, pituitary gland and testis tissues, 8 DEGs was identified in the comparison between hypothalamus and pituitary gland tissues, and 92 DEGs displayed differential expression between testis and external genitalia tissues, indicating different transcriptional changes in HPG axis during external genitalia development (Fig. 2b). The hierarchical clustering map (Fig. 2c-f) also recapitulated the distinct gene expression patterns in the hypothalamus, pituitary gland, testis and external genitalia between NEGG and AEGG.

\section{Functional enrichment analysis of the DEGs identified between NEGG and AEGG}

The results from $\mathrm{GO}$ enrichment analysis gave a more comprehensive understanding of the functions of the DEGs involved in the external genitalia development of male geese. The DEGs identified in the hypothalamus, pituitary gland, testis and external genitalia were annotated with $\mathrm{GO}$ database into three categories, including the biological process (BP), cellular component (CC), and molecular function (MF) (Additional file 3: Table S3). In the hypothalamus, most of DEGs were enriched in collagen trimer (CC), 
basement membrane (CC), and developmental process (BP) (Corrected $p<0.05)$ (Fig. 3a). In the pituitary gland, most of DEGs were enriched in integral component of plasma membrane (CC), neuron projection (CC), signal transduction (BP), extracellular matrix (CC), and calcium ion binding (MF) (Corrected $p<0.05$ ) (Fig. 3b). In the testis, most of DEGs were enriched in microtubule-based movement (BP), microtubule binding (MF), flagellated sperm motility (BP), mitotic cytokinesis (BP), neuropeptide signaling pathway (BP), microtubule motor activity (MF), and integral component of plasma membrane (CC) (Corrected $p<$ 0.05) (Fig. 3c). In the external genitalia, most of DEGs were enriched in integral component of membrane (CC), extracellular space (CC), plasma membrane (CC), cell surface (CC), calcium ion binding (MF), integral component of plasma membrane (CC), cell differentiation (BP), cell surface receptor signaling pathway (BP), and canonical WNT signaling pathway (Corrected $p<0.01)$ (Fig. 3d).

Subsequently, KEGG enrichment analysis showed that a total of $23,56,104$, and 99 KEGG pathways were enriched in the hypothalamus, pituitary gland, testis, and external genitalia, respectively (Additional file 3 : Table S4). The top 20 significantly enriched KEGG pathways were listed in Fig. 4. In the hypothalamus, the most enriched KEGG pathways were ECM-receptor interaction, purine metabolism, and MAPK signaling pathway (Fig. 4a). In the pituitary gland, the five most enriched pathways were ECM-receptor interaction, focal adhesion, neuroactive ligand-receptor interaction, p53 signaling and GnRH signaling pathways (Fig. 4b). Most of the top enriched KEGG pathways were neuroactive ligand-receptor interaction, cell cycle, oocyte meiosis, purine metabolism, progesterone-mediated oocyte maturation, calcium signaling, and WNT signaling pathways in the testis (Fig. 4c). In the external genitalia, the seven most enriched pathways were metabolic, neuroactive ligand-receptor interaction, calcium signaling, WNT signaling, adrenergic signaling in cardiomyocytes, ECM-receptor interaction, and purine metabolism pathways (Fig. 4d). Notably, the neuroactive ligand-receptor interaction pathway was significantly coenriched by the DEGs in the pituitary gland, testis, and external genitalia between NEGG and AEGG.

\section{Construction of co-expression networks of the DEGs identified between NEGG and AEGG}

WGCNA was performed to construct the co-expression networks of DEGs in the hypothalamus, pituitary gland, testis and external genitalia between NEGG and AEGG. The genes with similar expression patterns were classified into different modules (Fig. 5a) (Additional file 3: Table S5). Then, correlation analysis between the modules and phenotypes showed that the blue and brown module had a strong correlation with external genitalia development (Fig. 5b). Meanwhile, correlation analysis between these modules was also conducted and the results indicated that the gene expression pattern in blue module was similar to that in brown module (Fig. 5c, d). Thus, genes in the blue and brown modules were selected for further analysis.

\section{Network analyses of the DEGs involved in regulating external genitalia development of male geese}

To further identify the hub genes that were associated with external genitalia development, the DEGs from the hypothalamus, pituitary gland, testis and external genitalia between NEGG and AEGG were merged to construct the PPI network (Fig. 6a). The PPI network consisted of 133 nodes and 1531 edges. Subsequently, functional analyses revealed that the PPI network were significantly enriched in two 
pathways ("neuroactive ligand-receptor interaction" and "WNT signaling" pathways). The top highest degree genes included KNG1, LPAR2, LPAR3, NPY, PLCB1, AVPR1B, GHSR, GRM3, HTR5A, FSHB, FSHR, WNT11, WNT5A, WIF1, and WNT7B. Significantly, we found the "neuroactive ligand-receptor interaction" pathway regulated the "Wnt signaling" pathway through $P L C B 1$ to control the development of male goose external genitalia (Fig. 6b).

\section{Discussion}

In birds, the HPG axis is a coordinated neuroendocrine system whose function is vital for the regulation of reproductive system development $[18,19]$. A previous study has shown that male external genitalia development is significantly regulated by many types of hormones [20]. In the present study, a significant difference in the length and weight of the external genitalia between the NEGG and AEGG groups was observed. Consistent with this observation, 107, 284, 2192, and 1005 DEGs were identified in the hypothalamus, pituitary gland, testis and external genitalia between NEGG and AEGG, respectively. Recently, transcriptome studies in poultry HPG axis-related tissues have identified some candidate genes associated with the reproductive system development $[17,21]$. Taken together, these identified DEGs can potentially be used to explain the specific functions of HPG axis regulating the development of external genitalia of male goose.

To further reveal the biological implications of these identified DEGs, GO annotation and KEGG enrichment analysis were performed. Most of the DEGs in the hypothalamus were more enriched in GO terms related to collagen trimer, basement membrane, visual perception, and developmental process, suggesting that structural components and developmental process could be essential for the external genitalia development of male geese. The DEGs in the pituitary gland were mainly enriched in integral component of plasma membrane, neuron projection, signal transduction, extracellular matrix, collagencontaining extracellular matrix, extracellular space, calcium ion binding, and heparin binding. Several DEGs including FSHB, HPCA, NDOR1, KCNJ5, and GPD1L have been reported to be associated with reproductive performance. Expression levels of $F S H B$ were closely related to the serum levels of some reproductive hormones and on gonadal development in male [22, 23]. KEGG analysis showed that the DEGs identified in the pituitary gland between NEGG and AEGG were mainly involved in signaling pathways such as ECM-receptor interaction, focal adhesion, neuroactive ligand-receptor interaction, MAPK signaling, cytokine-cytokine receptor interaction, p53 signaling and GnRH signaling pathways. In chickens, the neuroactive ligand-receptor interaction signaling pathway recently been examined in ovaries where it regulates sexual maturity and ovary development [24].

Most of the DEGs in the testis were enriched in GO terms related to the microtubule-based movement, microtubule binding, flagellated sperm motility, mitotic cytokinesis, neuropeptide signaling pathway, microtubule motor activity, and integral component of plasma membrane. Several DEGs including FOXA2, SIX2, SNTN, VSTM2A, TDGF1, APOB, and WNT6 were found to be up-regulated in AEGG geese. Moreover, the DEGs in the testis were mainly enriched into 7 KEGG pathways, including neuroactive ligand-receptor interaction, cell cycle, purine metabolism, oocyte meiosis, calcium signaling pathway, 
WNT signaling pathway, and progesterone-mediated oocyte maturation, which were important for the external genitalia development. Previous studies have shown that the cell cycle and WNT signaling pathway played an important role in the regulation of mammalian genitalia development $[25,26]$.

In the external genitalia, most of DEGs were enriched in integral component of membrane, extracellular space, plasma membrane, cell surface, calcium ion binding, integral component of plasma membrane, and cell differentiation. Numerous studies have shown that cell cycle and cell differentiation play an important role in the development of external genitalia [25, 27]. Expression levels of $B M P 3, H S P A 2, S K I 1$, WNT9A, AGRN, WNT5A, WNT7B, CHRM3, MSTN, FSHR, and LHCGR genes were involved in the development of genitalia. The DEGs in the external genitalia were mainly enriched into metabolic, neuroactive ligand-receptor interaction, calcium signaling, WNT signaling, adrenergic signaling in cardiomyocytes, ECM-receptor interaction, and purine metabolism pathways. Previous studies have shown that $B m p$ gene could negatively affect proximodistally oriented outgrowth of external genitalia tubercle with regulatory functions on cell proliferation and apoptosis [27]. Moreover, expression of the Wnt signaling pathway related genes (WNT5A, WNT7A, and WNT9A) expression are regulated by both estrogen and androgen to direct the proliferation and elongation of the genitalia during differentiation [26].

In addition, the co-expression network analysis results suggested that "neuroactive ligand-receptor interaction" and "WNT signaling" pathways could play a critical role in affecting male goose external genitalia development. In the present study, the neuroactive ligand-receptor interaction pathway was significantly enriched by both the DEGs identified in the pituitary gland, testis and external genitalia between NEGG and AEGG groups. Transcriptome studies in poultry [16], goat [28], and yellow croaker fish [29] have also demonstrated the important role of this pathway in the control of reproductive activities. Furthermore, almost all DEGs enriched in this pathway, including KNG1, LPAR2, LPAR3, NPY, AVPR1B, GHSR, GRM3, HTR5A, FSHB, and FSHR, were significantly up- or down-regulated in the pituitary gland, testis and external genitalia between NEGG and AEGG groups. As we know, FSHB, FSHR, and GHSR are directly related to hormonal synthesis. $L P A R 3$ could interact with multiple reproductive hormones, including progesterone [30], and estrogen [31], to affect reproduction performance. Previous reports has shown that altering estrogen levels in males led to abnormal genitalia phenotypes $[8,26]$. Next, we found that the WNT signaling pathway likely played a significant role during external genitalia development in male geese. In this pathway, PLCB1, WIF1, WNT5A, WNT11, and WNT7B were downregulated in the AEGG group. Additionally, we found the neuroactive ligand-receptor interaction pathway could regulate the WNT signaling pathway through PLCB1. In recent years, studies in male have also demonstrated the important role of the WNT signaling pathway in the control of genitalia development [32-34]. During external genitalia development, the expression levels of WNT5A, WNT7A, and WNT9A were tightly regulated by hormones during external genitalia differentiation and are critical for its development [26]. As a WNT inhibitory factor, WIF1 was shown to inhibit the WNT / $\beta$-catenin signaling pathway by binding to WNT molecules to regulate the development of external genitalia [35]. Taken together, our results suggested that the neuroactive ligand-receptor interaction pathway may regulate the WNT signaling pathway through PLCB1 to control the external genitalia development of male goose. 


\section{Conclusions}

In conclusion, the genome-wide transcriptomic profiles in the hypothalamus, pituitary gland, testis, and external genitalia between the NEGG and AEGG groups were compared using RNA-sEq. Comprehensive analysis suggested that these identified DEGs in both the neuroactive ligand-receptor interaction and WNT signaling pathways were crucial for the external genitalia development. Furthermore, we found that the neuroactive ligand-receptor interaction pathway may regulate the WNT signaling pathway through PLCB1 to control the development of the male goose external genitalia. These results provide novel insights into the mechanisms regulating the external genitalia development of male goose.

\section{Methods}

\section{Experiment animals and sample collection}

The NEGG and AEGG geese were obtained from the Sichuan Agricultural University Waterfowls Breeding Farm (Ya'an, Sichuan, China). All of these geese were provided with free access to feed and water under natural light and temperature condition. At the age of 245 days, the external genitalia length was measured from the top of the external genitalia to the anus using a vernier caliper. When the length of the external genitalia was greater than $4.07 \mathrm{~cm}$, male individuals were classified into the normal external genital group (NEGG); in contrast, those with the external genitalia length less than $2.21 \mathrm{~cm}$ were classified into the abnormal external genitalia group (AEGG). In each group, three geese, with similar body weights and physiology conditions were selected for tissue sample collection. All selected geese were euthanized by inhaling carbon dioxide and cervical dislocation, which performed by competent personnel who experienced and correctly applied the technique. Then, the hypothalamus, pituitary gland, testis and external genitalia removed immediately after slaughter. Testis and external genitalia weights were collected quickly. All tissues were washed with PBS and frozen in liquid nitrogen, and then stored at $-80^{\circ} \mathrm{C}$ until RNA extraction.

\section{RNA isolation and sequencing}

For total RNA sequencing, total RNA from hypothalamus, pituitary gland, testis and external genitalia for each individual (a total of 24 samples) were extracted with the using RNeasy Mini Kit (QIAGEN, Beijing, China) following the manufacturer's instructions. RNA integrity was checked by Agilent Bioanalyzer 2100 (Agilent Technologies, Santa Clara, CA, USA). Samples with average RIN value of 8.96 (from 7.9 to 9.7) were then sent to generate libraries by Novogene (Novogene, Tianjin, China) (Additional file 3: Table S1). All libraries were sequenced by the Novogene Illumina PE 150. The clean reads were obtained after the filtration of low-quality reads using standard quality control by FastaQC software.

\section{Transcriptome alignment and assembly}

Clean reads were mapped against goose reference genome that includes the mitochondrial genome (AnsCyg_PRJ-NA183603_v1.0) using the HISAT2 (version 2.1.0) software [36]. The output SAM 
(sequencing alignment/mapping) file was converted to a BAM (binary alignment/mapping) file and sorted using SAMtools (version 1.13) [37]. The expression values (fragments per kilobase of transcript per million fragments mapped) of each gene were calculated based on the length of the gene and the read count mapped to this gene by featureCounts (version 2.02) [38].

\section{Identification of the DEGs and functions analysis}

According to the above groups (NEGG and AEGG) based on the length of external genitalia, we performed normalization and differential gene expression analysis using the DESeq2 [39]. False Discovery Rate (FDR) was used as corrected $p$-values $(\leq 0.05)$ due to multiple analysis. The DEGs was filtered based on $p$-values $<0.05$ and $|\log 2 \mathrm{FC}|>1$. KOBAS 3.0 [40]was used to analyze the Gene Ontology (GO) functions and the Kyoto Encyclopedia of Genes and Genomes (KEGG) functions. The weighted gene co-expression network analysis (WGCNA) package in R was used to construct the co- expression network for all genes in different tissues [41]. The STRING 10 database (http://string-db.org/) was employed to identify the relationship between the candidate genes identified in this study. All the network visualization was performed using Cytoscape (version 3.2.1) [42].

\section{List Of Abbreviations}

DEGs, differentially expressed genes; NEGG, normal external genital group; AEGG, abnormal external genitalia group; FDR, False Discovery Rate; GO, Gene Ontology; KEGG, Kyoto Encyclopedia of Genes and Genomes; WGCNA, weighted gene co-expression network analysis; PPI, protein-protein interaction; Shh, sonic hedgehog; IGF1, insulin growth factor $1 ; B m p 4$, bone morphogenetic protein 4; CYP17, 17ahydroxylase/17, 20-lyase.

\section{Declarations}

\section{Ethics approval and consent to participate}

All animal handling procedures were approved by the Institutional Animal Care and Use Committee (IACUC) of Sichuan Agricultural University (Chengdu campus, Sichuan, China, Permit No. DKY20170913). All methods were carried out in accordance with the ARRIVE guidelines (AVMA Guidelines for the Euthanasia of Animals: 2020 Edition).

\section{Consent of publication}

Not applicable

\section{Availability of data and materials}

All data generated or analyzed during this study are included in this published article and its additional files, or in the following public repositories. The transcriptome sequence data has been submitted to 
the NCBI Sequence Read Archive repository under the following accession numbers: [PRJNA764457] (https://www.ncbi.nlm.nih.gov/bioproject/764457).

\section{Competing interests}

The authors declare that they have no competing interests.

\section{Funding}

This study was supported by the China Agricultural Research System of MOF and MARA (CARS-42-4) and Key Technology Support Program of Sichuan Province (2021YFYZ0014).

\section{Author's contributions}

BCT, SQH and JWW designed the project with major contributions from QYOY and THW for the data analysis. YL and BCT collected the samples. JWH and BH provided the experimental animal. LL, JWH and JWW supervised the project. The manuscript was prepared by BCT and substantially revised by SQH. All authors read and approved the final manuscript.

\section{Acknowledgements}

Not applicable

\section{References}

1. Herrera AM, Shuster SG, Perriton CL, Cohn MJ. Developmental basis of phallus reduction during bird evolution. Current Biology. 2013;23(12):1065-1074.

2. Brennan P, Birkhead TR, Zyskowski K, Waag J, Prum RO. Independent evolutionary reductions of the phallus in basal birds. Journal of Avian Biology. 2008;39(5):487-492.

3. Akester AR. Form and Function in Birds. Journal of Anatomy. 1987;1:288-289.

4. Sanger TJ, Gredler ML, Cohn MJ. Resurrecting embryos of the tuatara, Sphenodon punctatus, to resolve vertebrate phallus evolution. Biology letters. 2015;11(10):20150694.

5. Coker CR, Frank MK, Helen H, Briggs SV, Cheng KM. Intromittent Organ Morphology and Testis Size in Relation to Mating System in Waterfowl. The Auk. 2002;(2):2.

6. House CM, Lewis Z, Hodgson DJ, Wedell N, Sharma MD, Hunt J, Hosken DJ. Sexual and natural selection both influence male genital evolution. PLoS One. 2013;8(5):e63807.

7. Perriton CL, Powles N, Chiang C, Maconochie MK, Cohn MJ. Sonic hedgehog signaling from the urethral epithelium controls external genital development. Developmental Biology. 2002;247(1):2646.

8. Chen Y, Yu H, Pask AJ, Fujiyama A, Renfree MB. Effects of androgen and oestrogen on the IGF pathways controlling phallus growth. Reproduction (Cambridge, England). 2018;157(1). 
9. Sun W, Gan X, Hu J, Li L, Wang J. CYP17 gene plays a key role in goose genital growth by influencing the testosterone level at puberty. Poult Science. 2018; 97(5):1748-1756.

10. Jia-Min, Jin, Wan-Xi, Yang. Molecular regulation of hypothalamus-pituitary-gonads axis in males. Gene. 2014;551(1):15-25.

11. Liu H, Wang J, Liang L, Han C, Xu H. Transcriptome analysis revealed the possible regulatory pathways initiating female geese broodiness within the hypothalamic-pituitary-gonadal axis. PLoS ONE. 2018;13(2):e0191213.

12. Nan W, Zhu Q, Chen B, Jian G, Li D. High-throughput sequencing of pituitary and hypothalamic microRNA transcriptome associated with high rate of egg production. Bmc Genomics. 2017;18(1).

13. Mishra SK, Chen B, Zhu Q, Xu Z, Li D. Transcriptome analysis reveals differentially expressed genes associated with high rates of egg production in chicken hypothalamic-pituitary-ovarian axis. Scientific Reports. 2020;10(1).

14. Li B, Fillmore N, Bai Y, Collins M, Thomson JA, Stewart R, Dewey CN. Evaluation of de novo transcriptome assemblies from RNA-Seq data. Genome Biology. 2014;15(12):553.

15. Stark R, Grzelak M, Hadfield J. RNA sequencing: the teenage years. Nature Reviews Genetics.

16. Ouyang Q, Hu S, Wang G, Hu J, Zhang J, Li L, Hu B, He H, Liu H, Xia L. Comparative Transcriptome Analysis Suggests Key Roles for 5-Hydroxytryptamlne Receptors in Control of Goose Egg Production. Genes. 2020;11(4):455.

17. Bai DP, Chen Y, Hu YQ, He WF, Li A. Transcriptome analysis of genes related to gonad differentiation and development in Muscovy ducks. BMC Genomics. 2020;21(1).

18. Leska A, Dusza L. Seasonal changes in the hypothalamo-pituitary-gonadal axis in birds. Reproductive biology. 2007;7(2):99-126.

19. Takayoshi U, Bentley GE, Kazuyoshi T. Neuroendocrine regulation of gonadotropin secretion in seasonally breeding birds. Frontiers in Neuroscience. 2013;7:38-.

20. Boas M, Boisen KA, Virtanen HE, Kaleva M, Suomi AM, Schmidt IM, Damgaard IN, Kai CM, Chellakooty M, Skakkebaek NE. Postnatal penile length and growth rate correlate to serum testosterone levels: a longitudinal study of 1962 normal boys. European Journal of Endocrinology. 2006;154(1):125-129.

21. Shiue YL, Chen LR, Chen CF, Chen YL, Ju JP, Chao CH, Lin YP, Kuo YM, Tang PC, Lee YP. Identification of transcripts related to high egg production in the chicken hypothalamus and pituitary gland. Theriogenology. 2006;66(5):1274-1283.

22. Tamburino L, Vignera SL, Tomaselli V, Condorelli RA, Mongioì L, Calogero AE. Impact of the FSHB gene - 211G/T polymorphism on male gonadal function. J Assist Reprod Genet. 2017;34(5):671676.

23. Horie Y, Shimizu A, Adachi S, Kobayashi T. Expression and localization of gonadotropic hormone subunits (Gpa, Fshb, and Lhb) in the pituitary during gonadal differentiation in medaka. General \& Comparative Endocrinology. 2014;204:173-180. 
24. Chen, Liang, Zhu, Tan, Liu. Gene Expression Profiling in Ovaries and Association Analyses Reveal HEP21 as a Candidate Gene for Sexual Maturity in Chickens. Animals. 2020;10(2):181.

25. Seifert AW, Zheng Z, Ormerod BK, Cohn MJ. Sonic hedgehog controls growth of external genitalia by regulating cell cycle kinetics. Nature Communications. 2010;1(3):1-9.

26. Chen Y, Yu H, Pask AJ, Asao F, Yutaka S, Sumio S, Geoff S, Renfree MB. Hormone-responsive genes in the $\mathrm{SHH}$ and WNT/ $\beta$-catenin signaling pathways influence urethral closure and phallus growth. Biology of Reproduction. 2018;(4):4.

27. Suzuki, K. Regulation of outgrowth and apoptosis for the terminal appendage: external genitalia: development by concerted actions of BMP signaling. Development. 2003;130(25):6209.

28. Su F, Guo X, Wang Y, Wang Y, Cao G, Jiang Y. Genome-Wide Analysis on the Landscape of Transcriptomes and Their Relationship With DNA Methylomes in the Hypothalamus Reveals Genes Related to Sexual Precocity in Jining Gray Goats. Frontiers in Endocrinology. 2018;9:501-.

29. Luo S, Gao X, Ding J, Liu C, Du C, Hou C, Zhu J, Lou B. Transcriptome Sequencing Reveals the Traits of Spermatogenesis and Testicular Development in Large Yellow Croaker (Larimichthys crocea). Genes. 2019;10(12).

30. Liszewska E, Reinaud P, Dubois O, Charpigny G. Lysophosphatidic acid receptors in ovine uterus during estrous cycle and early pregnancy and their regulation by progesterone. Domestic Animal Endocrinology. 2012;42(1):31-42.

31. Diao H, Li R, Zowalaty A, Xiao S, Zhao F, Dudley EA, Ye X. Deletion of Lysophosphatidic Acid Receptor 3 (Lpar3) Disrupts Fine Local Balance of Progesterone and Estrogen Signaling in Mouse Uterus During Implantation. Biology of Reproduction. 2015; 93(5):123-123.

32. Han SC, Szucsik JC, Georgas KM, Jones JL, Rumballe BA, Tang D, Grimmond SM, Lewis AG, Aronow BJ, Lessard JL. Comparative gene expression analysis of genital tubercle development reveals a putative appendicular Wnt7 network for the epidermal differentiation. Developmental Biology. 2010;344(2):1071-1087.

33. Shinichi M, Yoshihiko S, Ryuma H, Kentaro S, Taisen I, Taketo MM, Naomi N, Takahiro M, Ken-Ichi T, Shigeaki K. Genetic interactions of the androgen and Wnt/beta-catenin pathways for the masculinization of external genitalia. Molecular Endocrinology. 2009;(6):871-880.

34. Seifert AW, Yamaguchi T, Cohn MJ. Functional and phylogenetic analysis shows that Fgf8 is a marker of genital induction in mammals but is not required for external genital development. Development. 2009;136(15):2643.

35. Ng CL, Matsumaru D, Ho SH, Garcia-Barceló M, Yuan ZW, Smith D, Kodjabachian L, Tam KH, Yamada G, Lui CH. Dysregulation of Wnt inhibitory factor 1 (Wif1) expression resulted in aberrant Wnt- $\beta$ catenin signaling and cell death of the cloaca endoderm, and anorectal malformations. Cell Death \& Differentiation. 2014;21(6):978-989.

36. Kim D, Langmead B, Salzberg SL. HISAT: A fast spliced aligner with low memory requirements. Nature Methods. 2015;12(4). 
37. Li H, Handsaker B, Wysoker A, Fennell T, Ruan J, Homer N, Marth G, Abecasis G, Durbin R. The Sequence Alignment/Map format and SAMtools. Bioinformatics. 2009;25(16):2078-2079.

38. Yang L, Smyth GK, Wei S. featureCounts: an efficient general purpose program for assigning sequence reads to genomic features. Bioinformatics. 2014;(7):923-930.

39. Love MI, Huber W, Anders S. Moderated estimation of fold change and dispersion for RNA-seq data with DESeq2. Genome Biology. 2014;15(12):550.

40. Chen, Ai, Lei, Kong. CGPS:A machine learning-based approach integrating multiple gene set analysis tools for better prioritization of biologically relevant pathways. Journal of Genetics and Genomics. 2018.

41. Langfelder P, Horvath S. WGCNA: an R package for weighted correlation network analysis. Bmc Bioinformatics. 2008;9(1):559.

42. Smoot, Michael E, Ono, Keiichiro, Ruscheinski, Johannes, Wang, Peng-Liang, Ideker, Trey. Cytoscape 2.8: new features for data integration and network visualization. Bioinformatics. 2011.

\section{Figures}

\section{Figure 1}

a

b
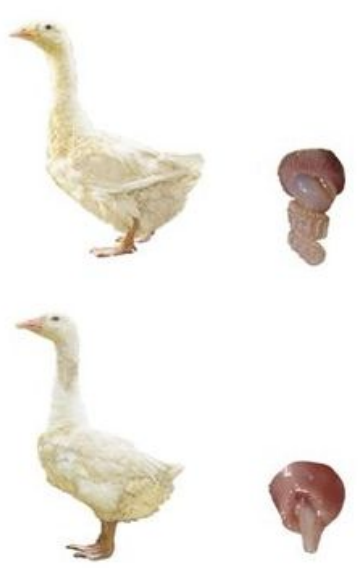

C

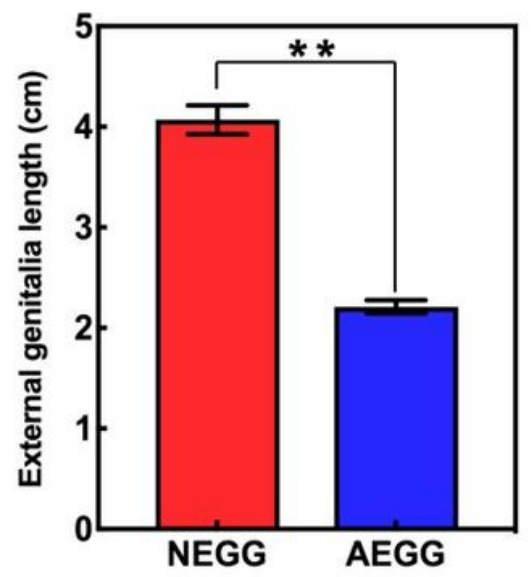

d

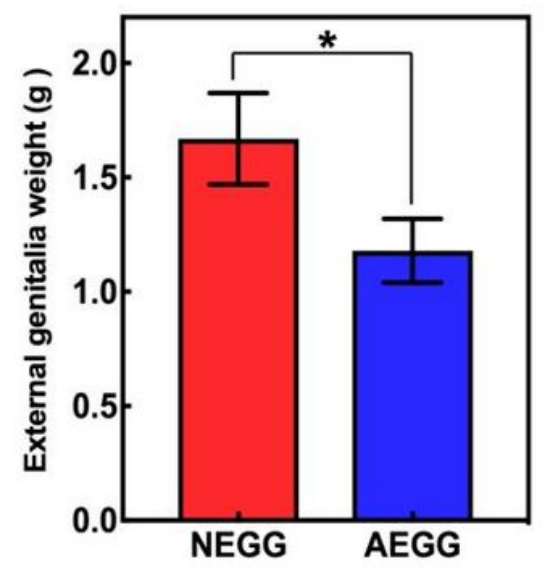

\section{Figure 1}

Morphological differences in the external genitalia between NEGG and AEGG geese. (a). Normal external genitalia group geese. (b). Abnormal external genitalia group geese. (c). The length of external genitalia. (d). The weight of external genitalia. 
Figure 2
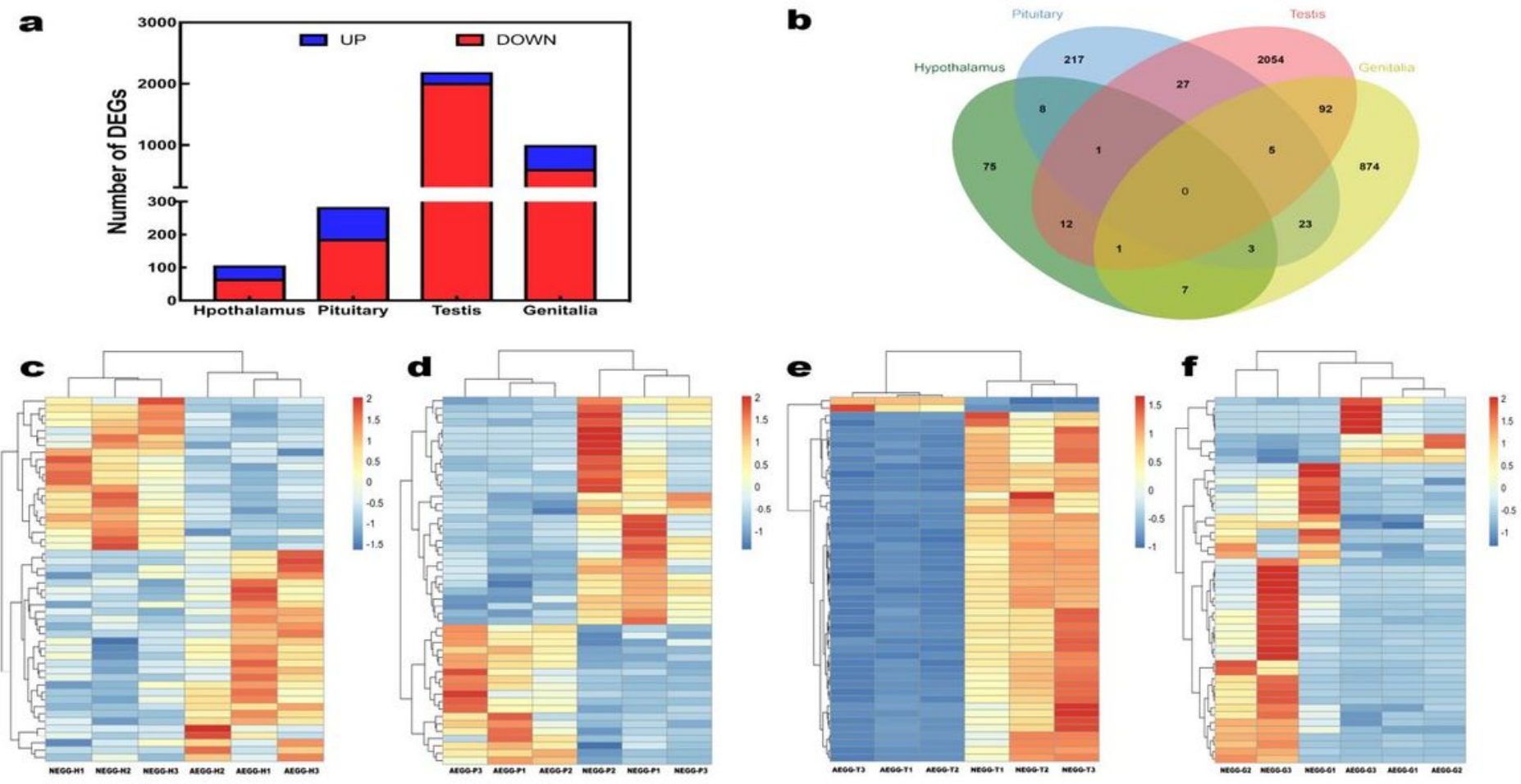

\section{Figure 2}

Transcriptomic changes for each tissue and each group. (a). The number of DEGs in different tissues. (b). Venn diagram of number of DEGs between NEGG and AEGG. (c-f). Pearson's correlation matrix for mRNA profiles. 


\section{Figure 3}

a

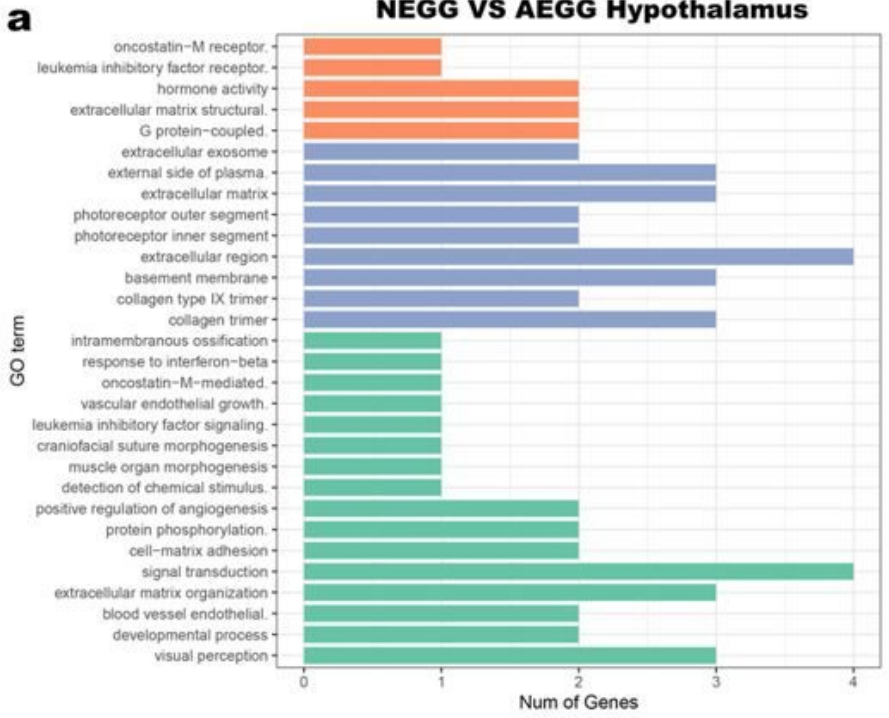

c

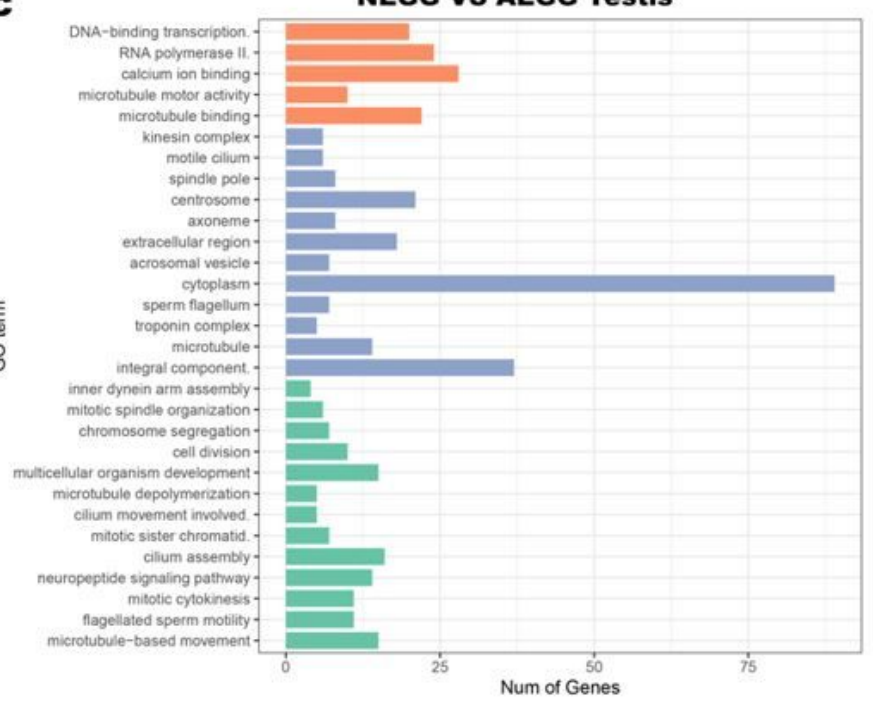

molecular_function

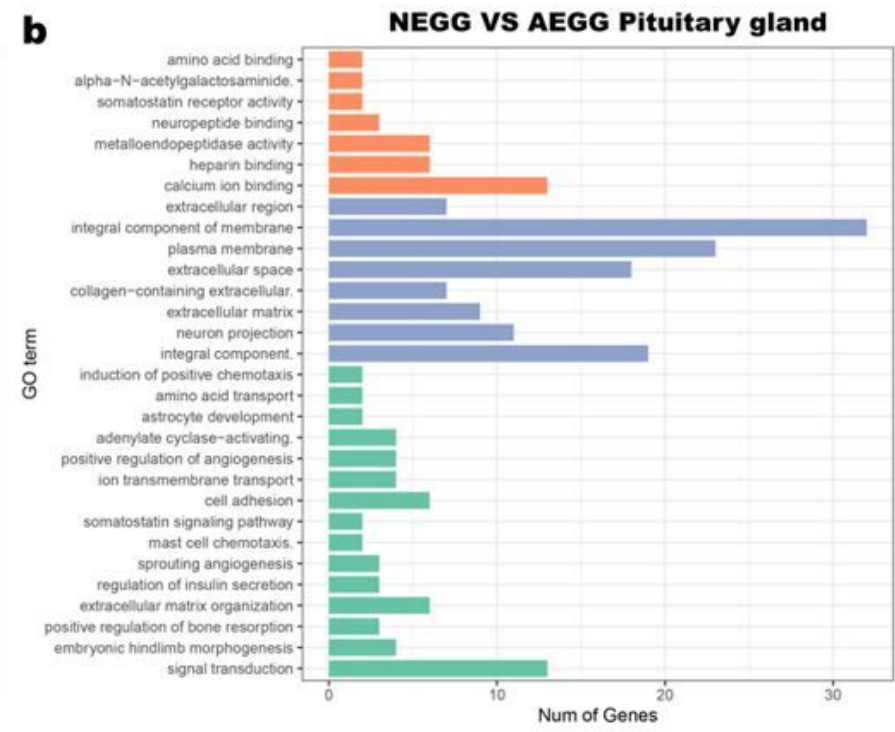

d

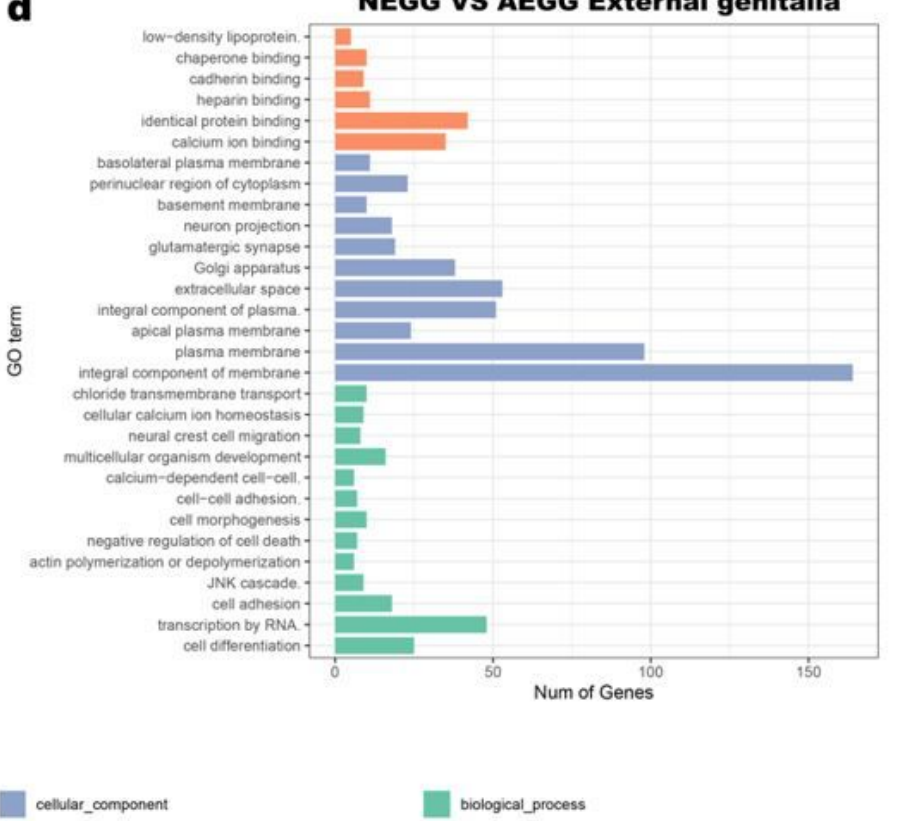

\section{Figure 3}

GO enrichment terms of DEGs. (a). NEGG-vs-AEGG hypothalamus; (b). NEGG-vs-AEGG pituitary gland; (c). NEGG-vs-AEGG testis; (d). NEGG-vs-AEGG external genitalia. The results are summarized in three main categories: biological process (BP), cellular component (CC) and molecular function (MF). The $x$-axis indicates the number of genes, and the y-axis indicates the second-level GO term. 


\section{Figure 4}
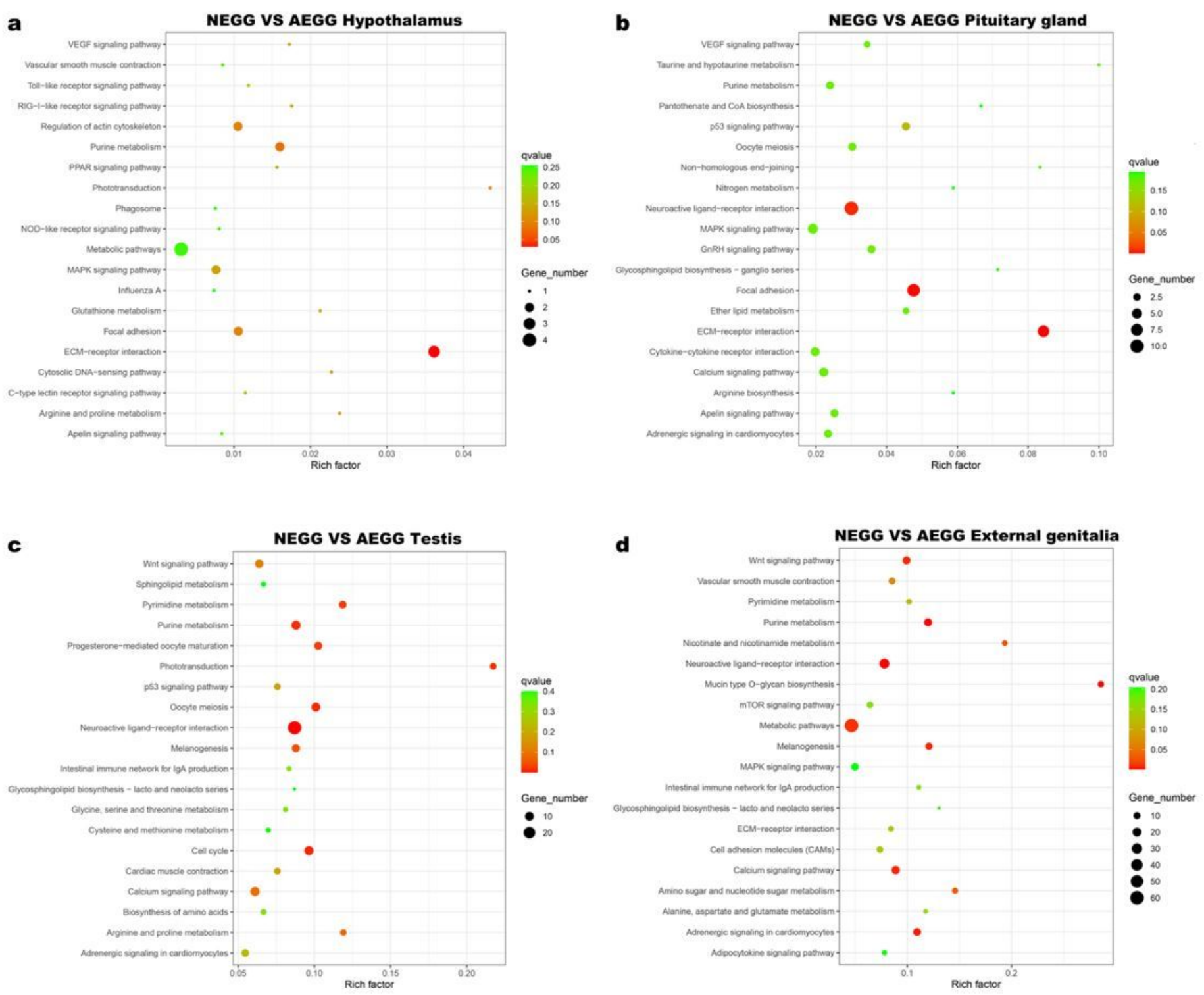

\section{Figure 4}

Top 20 pathways in KEGG enrichment by q-Value. (a). NEGG-vs-AEGG hypothalamus; (b). NEGG-vs-AEGG pituitary gland; (c). NEGG-vs-AEGG testis; (d). NEGG-vs-AEGG external genitalia. The Rich factor is the ratio of the number of DEGs in the pathway and the total number of genes in the pathway. The higher the Rich factor, the higher is the degree of enrichment. The q-Value is the P-value after multiple hypothesis test correction, in the range from 0 to 1 ; the closer the q-Value is to zero, the more significant is the enrichment. 


\section{Figure 5}

a

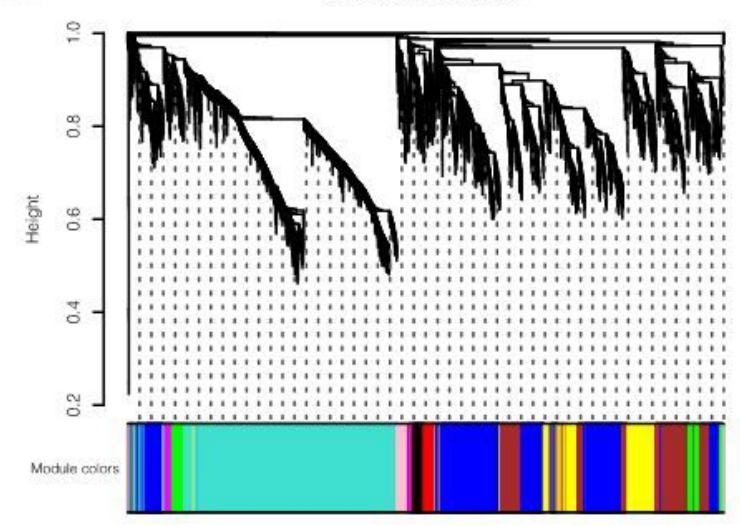

c

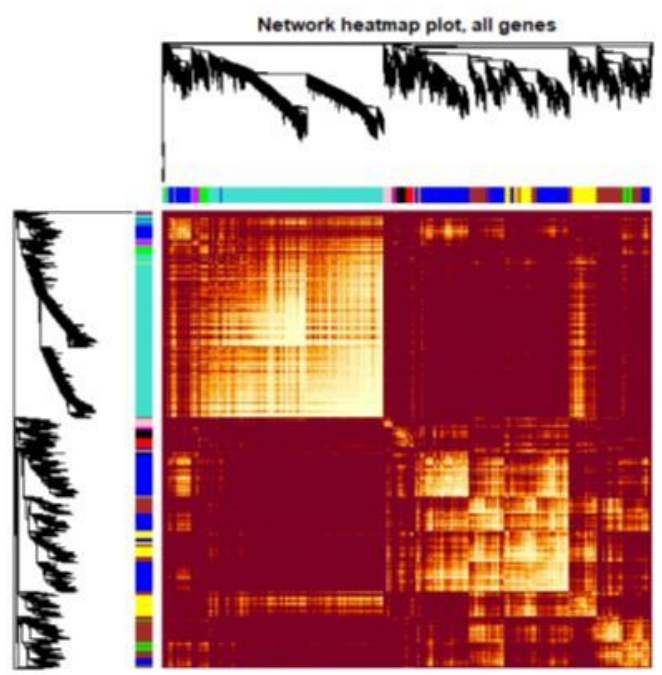

b Module-trait relationships

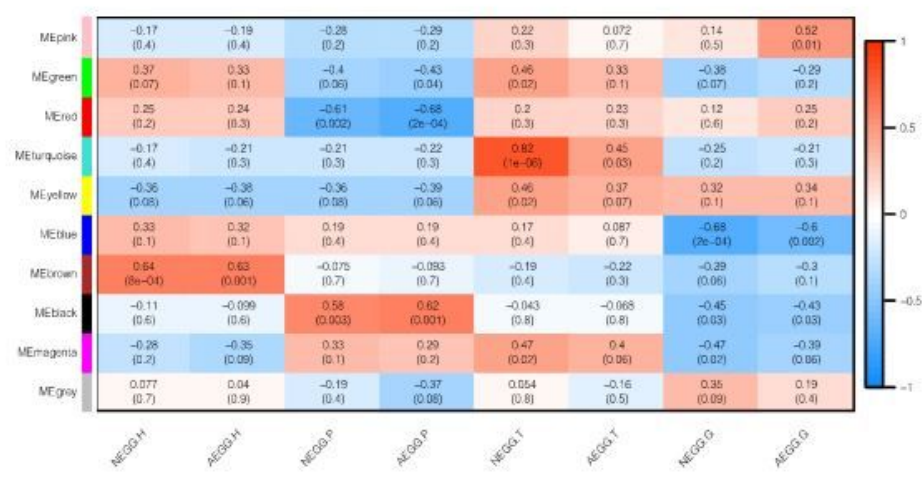

d

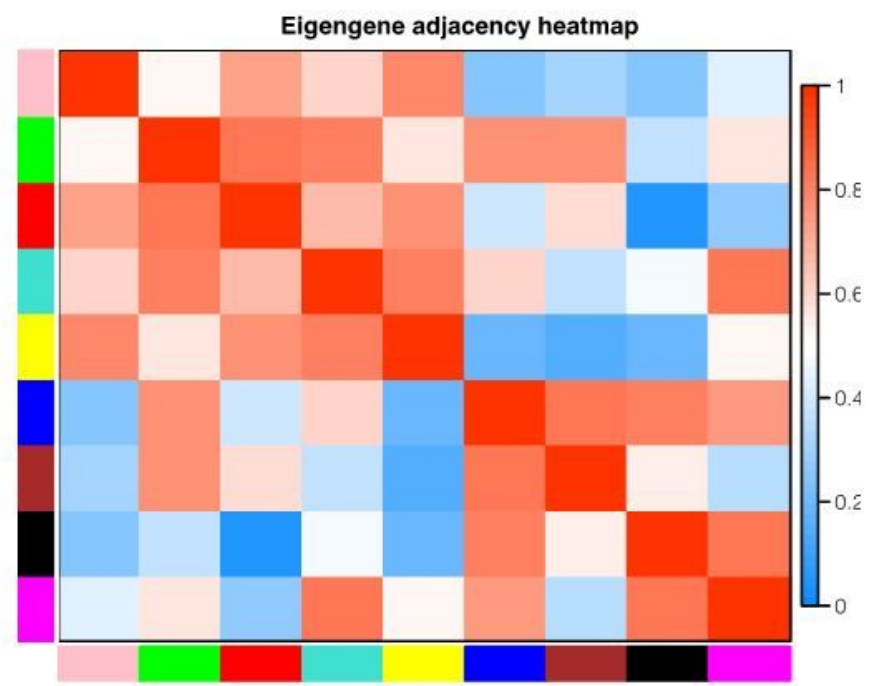

Figure 5

Weighted gene co-expression network analysis (WGCNA). (a). Hierarchical clustering tree. Different colors on the abscissa represent different clustering modules. (b). Correlation between modules and traits. The abscissa represents different trait groups, and the ordinate represents different modules. (c). Visualized network heat map. (d). Correlation diagrams between modules. The redder the color of the area where different modules intersect is, the stronger the correlation. 


\section{Figure 6}

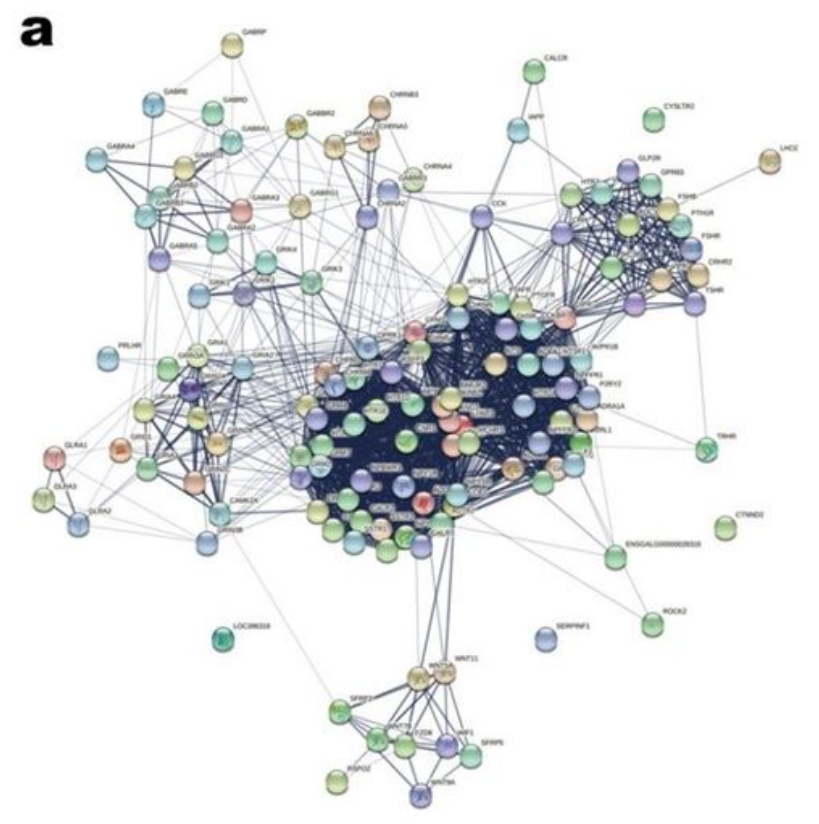

b

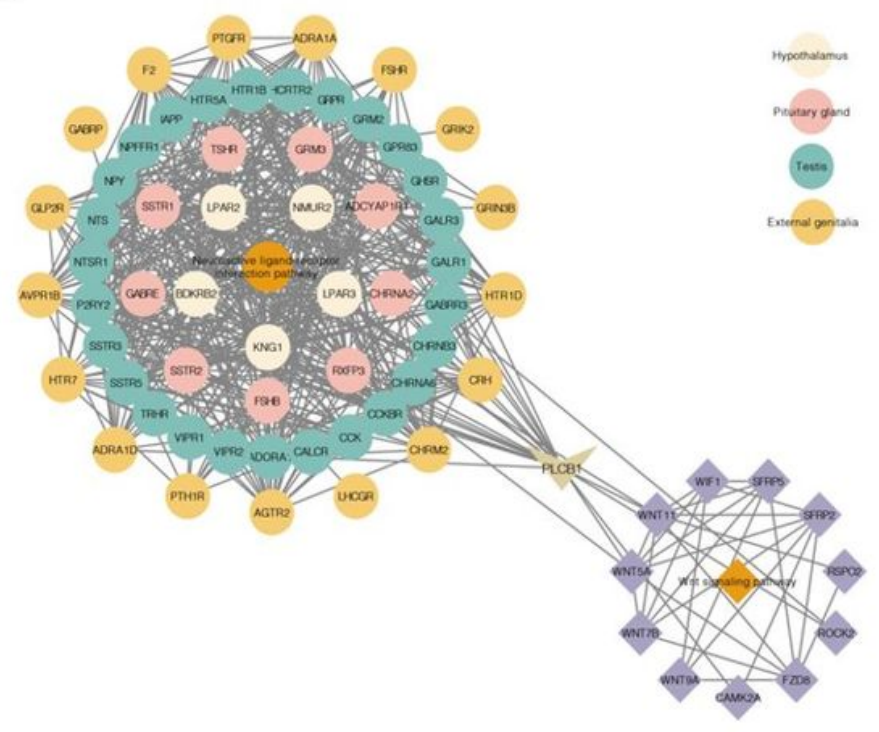

Figure 6

Network analyses for DEGs between NEGG and AEGG. (a). PPI networks of DEGs. The network was constructed by 136 DEGs based on fold change of DEGs, WGCNA, KEGG pathway, and GO enrichment, which consisted of 2 significantly important pathways. (b). Regulation network construction involved in external genitalia development of male geese. The circle and diamond represent the neuroactive ligandreceptor interaction pathway and WNT signaling pathway, respectively.

\section{Supplementary Files}

This is a list of supplementary files associated with this preprint. Click to download.

- Additionalfile1.xlsx

- Additionalfile2.xIsx

- Additionalfile3.xlsx

- Additionalfile4.xIsx

- Additionalfile5.xIsx 